\title{
Mapa Cultural do Ceará: Redesign a partir da avaliação de usabilidade e experiência do usuário
}

\author{
Patrick de Araújoํㅜ, Mateus Carneiro ${ }^{1}$, Ticianne Darin ${ }^{1}$ \\ 1Universidade Federal do Ceará \\ Fortaleza - Ceará - Brasil \\ patrick.nobre12@gmail.com, \{mateus, ticianne\} @virtual.ufc.br
}

\begin{abstract}
Digital collaborative maps are tools that allow the production and insertion of information about a space to be shared among users. One of these platforms is the Ceará Cultural Map, with numerous registered agents and very relevant to the local cultural scene. Thus, this work aimed to evaluate usability and user experience to enhance the collaborative aspect through a redesign. The work was carried out in four stages: (1) heuristic evaluation and usability tests using the MAX method; (2) Analysis and triangulation of data; (3) Application redesign; and (4) Redesign evaluation with usability tests and interviews. From this, it was found that the process carried out helped to think of visual and interaction solutions that seek to enhance the collaborative character of the interface.
\end{abstract}

Resumo. Os mapas colaborativos digitais são ferramentas que permitem a produção $e$ inserção de informações sobre um espaço para serem compartilhadas entre usuários. Uma dessas plataformas é o Mapa Cultural do Ceará, com grande número de agentes cadastrados e muito relevante para o cenário cultural local. Assim, este trabalho teve como objetivo realizar avaliação de usabilidade e experiência do usuário para potencializar o aspecto colaborativo através de um redesign. O trabalho foi realizado em quatro etapas: (1) avaliação heurística e testes de usabilidade com método MAX; (2) Análise e triangulação dos dados; (3) Redesign da aplicação; e (4) Avaliação do redesign com testes de usabilidade e entrevista. A partir disso, constatou-se que o processo realizado ajudou a pensar em soluções visuais e de interação que buscam potencializar o caráter colaborativo da interface.

\section{Introdução}

As novas tecnologias permitiram que meios de comunicação mais interativos surgissem, em que limitações de espaço e tempo são desconsideradas e que qualquer pessoa possa acessar de forma rápida uma informação ou manter contato com pessoas distantes. Destaca-se também que as ferramentas de comunicação pela internet funcionam de modo que uma pessoa produz e/ou consome conteúdo [Vermelho et al. 2014]. .

Com essas características, os mapas colaborativos são instrumentos que propiciam experiências de relação dos usuários com esquemas de dimensões territoriais e localização espacial em formato de mapa, viabilizando a inserção de dados personalizados junto a dados inseridos por outros usuários para a criação de um repositório de informações compartilhadas de forma voluntária [Ribeiro e Lima 2011]. $\mathrm{O}$ desenvolvimento de plataformas desse tipo na área da cultura começou no Brasil a partir de 2004, com o surgimento do software livre Mapsys. A partir disso, surgiram iniciativas de mapeamento como o Pontos de Cultura do Ministério da Cultura em 2005 e o portal CulturaEduca pelo Instituto Lidas em 2010, até a criação do projeto Mapas 
Culturais, em 2014, parceria do Instituto Tim com a Secretaria Municipal de Cultura de São Paulo [Rigolon 2016].

O Mapa Cultural do Ceará é uma plataforma digital colaborativa desenvolvida pela Secretaria de Cultura do Ceará, em 2015, baseada no projeto Mapas Culturais, tendo como intuito reunir informações a respeito de projetos, eventos e espaços culturais em geral. [Rigolon et al. 2018]. Reconhece-se então a importância da plataforma ao se considerar que ela é feita por usuários colaboradores, que participam ativamente na construção de informações que possam servir de direcionamento de políticas públicas na cultura, além de contribuir para o conhecimento cultural cearense e também ser um meio de divulgação de oportunidades para os agentes. A relevância do Mapa Cultural do Ceará no cenário cultural local, que hoje conta com um número de 90.731 agentes culturais cadastrados (como são chamadas as pessoas cadastradas na plataforma) [Mapa Cultural do Ceará 2015], e as ações colaborativas dos usuários como visualizar, adicionar, editar e compartilhar informações são as principais motivações deste trabalho. Assim, o objetivo é realizar uma avaliação com usuários, ao analisar usabilidade e experiência do usuário, para potencializar o aspecto colaborativo da plataforma.

Este trabalho está estruturado em trabalhos relacionados, metodologia, resultados, discussão e conclusão. Apresenta processo de redesign do Mapa Cultural do Ceará por meio de avaliação por inspeção, testes de usabilidade e aplicação de métodos que se baseiam nas falas dos utilizadores. Com isso, ressalta-se também a potencial contribuição deste trabalho em servir de base para gerar mudanças em outros sistemas que utilizam a base do projeto Mapas Culturais.

\section{Trabalhos Relacionados}

Existem diversas plataformas de mapeamento colaborativo, entretanto, para a proposição de ferramentas desse tipo, deve-se considerar questões como objetivos do mapeamento, área de abrangência, o público, a plataforma a ser desenvolvida ou customizada e definição de categorias em relação ao temas e objetivos da plataforma [Nascimento 2015].

Nesse contexto, alguns estudos foram encontrados, como o trabalho de Castro et al. (2020) teve como objetivo desenvolver e avaliar um mapa interativo e colaborativo para ser disponibilizado como open source. A metodologia utilizada consiste em quatros etapas: análise bibliográfica sobre mapas interativos; desenvolvimento da solução na forma de uma aplicação web; avaliação comparativa da solução com outras iniciativas de mapeamento a partir do Modelo 4C, que tem como critérios a comunicação, coordenação, colaboração e cooperação; e avaliação de UX (por escala de diferencial semântico), Usabilidade (por meio de testes de usabilidade) e de Percepção de Valor (através de questionário). E de forma similar, Steil (2018) investigou a plataforma colaborativa RedeParaná em questões de satisfação e usabilidade, focando também em ambientes de compartilhamento e cultura de colaboração. Para isto, foram realizadas revisão bibliográfica, entrevistas, surveys e testes com usuários. Os dados triangulados foram utilizados para a definição de requisitos para um Redesign da plataforma.

Ambos os trabalhos se debruçam sobre avaliação, redesign e desenvolvimento de plataformas colaborativas, assim como o trabalho apresentado neste artigo, realizando 
propostas metodológicas para que designers e pesquisadores tenham uma base para aplicá-las. Assim, considerando o elemento participativo de usuários em plataformas colaborativas, este artigo se baseia no modelo hedônico/pragmático [Hassenzahl 2008], em que a qualidade hedônica é associada ao "eu", às necessidades humanas como novidade, autoexpressão, mudança etc., aspectos que contribuem para gerar experiências positivas e contribuir para a participação dos usuários, e a qualidade pragmática se relaciona a como esses usuários irão utilizar essas plataformas, considerando aspectos como funcionalidade, utilidade e usabilidade.

\section{Metodologia}

Este trabalho tem como objetivo a avaliação e o redesign do Mapa Cultural do Ceará ${ }^{1}$, para potencializar o seu aspecto colaborativo. Foi dividido em quatro etapas e foi conduzido em dois períodos principais: de janeiro a março de 2020 e de outubro de 2020 a agosto de 2021, considerando a versão da plataforma vigente nestes períodos $\mathrm{O}$ processo foi baseado no design de interação e as avaliações foram planejadas utilizando o framework DECIDE, que estrutura uma avaliação em seis etapas: Determine, Explore, Choose, Identify, Decide e Evaluate [Preece, Rogers e Sharp 2005].

A primeira etapa caracterizou-se por avaliação heurística. Esta etapa foi realizada por três avaliadores independentes que seguiram um roteiro ${ }^{2}$ e que teve como base a lista de 14 heurísticas de Pribeanu (2017), lista que integra as heurísticas de Nielsen aos critérios ergonômicos de Scapin e Bastien [Pribeanu 2017]. A escolha desta lista foi baseada na hierarquização das heurísticas, dispostas em grupos, facilitando questões de análise e de explicação dos dados. Cada avaliador realizou individualmente a inspeção e os dados encontrados foram consolidados por reunião remota, culminando numa lista final de problemas. Os dados em ambos os métodos foram registrados em documentos de texto e planilhas no Google Docs.

$\mathrm{Na}$ segunda etapa, foram realizados testes de usabilidade com dez usuários divididos igualmente em dois perfis: os agentes culturais (60\% homens e $40 \%$ mulheres) e o público em geral (60\% homens e $40 \%$ mulheres). Os usuários foram contatados de forma presencial e por meio de aplicativos de mensagem, em que a seleção dos agentes culturais se deu por consulta da atividade que exerciam e se já haviam utilizado a plataforma. Esses testes se estruturam num roteiro de cinco atividades ${ }^{3}$, em que sete sessões ocorreram de forma presencial em laboratório com a participação de dois avaliadores, anterior à pandemia de COVID-19, e três foram remotos com um avaliador, pelo serviço Google Meet e gravação por meio de programa externo, devido às medidas de distanciamento social. Após cada teste foi utilizado também o MAX (Method for the Assessment of eXperience) [Cavalcante, Rivero e Conte 2015], para avaliar a experiência dos usuários após utilizarem o sistema, através de cartas que representam os sentimentos destes, dispostas em um quadro com perguntas específicas. Esse método também foi adaptado para ser utilizado de forma remota, através da plataforma colaborativa de quadros em branco Miro.

\footnotetext{
${ }^{1}$ https://mapacultural.secult.ce.gov.br

${ }^{2}$ https://bit.ly/3tIUBko

${ }^{3}$ https://bit.ly/3zfjkhs
} 
Nesta etapa também realizaram-se análises dos dados gerados na etapa anterior. Os dados decorrentes da avaliação heurística foram analisados quantificando o total de problemas achados, problemas por heurística e problemas por grau de severidade. A análise qualitativa dos dados gerados se deu por meio da identificação de padrões que se repetissem nas falas dos usuários e registros anotados, além de também se registrarem sugestões [Preece, Rogers e Sharp 2005]. Após isso, foi realizado cruzamento de dados dos testes, da avaliação heurística e do MAX, novamente identificando questões recorrentes e únicas nos três métodos a fim de gerar uma lista de problemas a serem resolvidos.

$\mathrm{Na}$ terceira etapa, com base nos resultados das fases anteriores, foi feito o redesign da aplicação e obteve-se como resultado um protótipo navegável de alta fidelidade com a nova proposta de interface do Mapa Cultural do Ceará. Na última etapa, realizou-se a avaliação do redesign. Desta vez, devido a limitações de tempo para término do projeto, optou-se por realizar observação do uso com o protótipo interativo produzido e, em seguida, uma entrevista estruturada ${ }^{4}$, com todo o processo realizado remotamente através do Google Meet. Assim, participaram desta etapa 5 usuários (60\% homens e $40 \%$ mulheres), com experiência prévia de uso do Mapa e foram utilizadas as mesmas atividades da avaliação inicial. A entrevista, por sua vez, foi baseada nas perguntas estabelecidas pelo MAX, focando em aspectos de experiência do usuário. A análise foi feita da mesma forma que na terceira etapa buscando indicação de pontos positivos e negativos no redesign.

\section{Resultados}

\subsection{Avaliação inicial com o Mapa Cultural do Ceará}

A avaliação heurística ${ }^{5}$ foi um importante começo para entender sobre os problemas que o Mapa possuía. Foram encontrados 72 problemas, sendo 37,5\% (27) de severidade 2 (pequeno), 29,16\% (21) de severidade 3 (grande), 18,05\% (13) de severidade 4 (catastrófico) e 15,27\% (11) de severidade 1 (cosmético),

Dentre as heurísticas, destacam-se a de Prompting com 16.66\% (12) dos problemas (diz respeito a como guiar o usuário, mostrando o que ele deve fazer); a de Consistency com 13.88\% (10) (sobre providenciar decisões e significados similares em contextos parecidos); e a de Grouping/distinction com 12.5\% (9) (sobre agrupar elementos similares e explicitar diferenças entre elementos). Além destas, a heurística de Error Management merece destaque pois 5 dos 6 problemas identificados foram categorizados com severidade catastrófica. Os locais da plataforma em que os problemas foram encontrados em funcionalidades utilizadas para inserção ou edição de informações, meios pelos quais os usuários colaboram com a plataforma.

Em contrapartida, nos testes com usuários ${ }^{6}$ (realizados com dez pessoas) foi possível identificar que mesmo sem familiaridade com a plataforma, os usuários realizaram as atividades com sucesso. Os problemas encontrados refletem o que foi

\footnotetext{
${ }^{4}$ https://bit.ly/3nBoXV5

${ }^{5}$ https://bit.ly/3lvcFL4

${ }^{6}$ https://bit.ly/3tKVw3Z
} 
identificado na avaliação heurística (apesar de terem sido encontrados poucos problemas recorrentes entre os métodos). Além destas, importante também destacar como a seção de oportunidades (que agrega os editais) tiveram maior ocorrência de problemas que se repetiam entre os dois métodos utilizados.

Quanto aos resultados identificados pelas questões do $\mathrm{MAX}^{7}$, corrobora-se o que foi encontrado nos testes. Sobre os sentimentos ao utilizar a plataforma, 70\% (7) dos usuários se sentiram interessados e $40 \%$ também satisfeitos (4), mas ao mesmo tempo $50 \%$ (5) se sentiam perdidos e $20 \%$ (2) impacientes. Sobre a facilidade de uso, ocorre a mesma ambiguidade: $60 \%$ (6) dos usuários consideraram que foi fácil aprender a usar e $50 \%$ (5) entenderam como funciona, mas registraram ter cometido erros e que se perderam. Sobre a utilidade da plataforma, $80 \%$ (8) dos usuários consideraram que era útil e que lhes ajudaria $(60 \%, 7)$, e isso para diferentes finalidades. Quanto a atratividade do sistema, $50 \%$ (5) usuários têm uma percepção positiva ao indicarem que gostam do visual e gostam das cores, porém $40 \%$ (4) indicaram que não gostaram dos ícones e $30 \%$ (3) não gostaram dos componentes. Quanto ao desejo de usar, em que $70 \%$ (7) usariam novamente, pelo fato de terem que trabalhar com o Mapa, e 50\% (5) usariam se necessário; importante destacar também que $20 \%$ (2) usariam outro. Os principais problemas encontrados foram sintetizados na Tabela 1. Embora muitos deles não impeçam o usuário de utilizar a plataforma, afetam a experiência deles, dificultando o uso e a fidelização dos usuários.

Tabela 1. Principais problemas catalogados a partir de aplicação dos três métodos

\begin{tabular}{|c|l|}
\hline Cód. do Problema & \multicolumn{1}{|c|}{ Descrição } \\
\hline P01 & Carência em informar devidamente status do sistema \\
\hline P02 & Botões e elementos mal agrupados \\
\hline P03 & $\begin{array}{l}\text { Elemento gráficos (interativos ou não) pequenos, com pouco } \\
\text { destaque ou não nomeados, que tem função de guiar os usuários }\end{array}$ \\
\hline P04 & Ausência de botões de adicionar na visão em mapa \\
\hline P05 & $\begin{array}{l}\text { Formulários com muitos campos (ao adicionar ou editar eventos, } \\
\text { espaços, projetos ou agentes e se inscrever em oportunidade) }\end{array}$ \\
\hline P06 & Página inicial com informações desnecessárias e textos longos \\
\hline P07 & Ausência de confirmação para prevenção de erros \\
\hline P08 & Título de algumas seções e pouca clareza em diferenciá-los \\
\hline P09 & Aplicação das cores e contrastes de textos com fundo \\
\hline P10 & Visual (leiaute, ícones e forma de elementos gráficos em geral) \\
\hline
\end{tabular}

\subsection{Redesign do Mapa Cultural do Ceará}

No protótipo interativo criado $^{8}$ após a avaliação optou-se por não fazer mudanças de fluxo de interação para valorizar o conhecimento prévio dos usuários. Foram incorporadas soluções para potencializar as melhorias advindas dos problemas descritos na Tabela 1. A Figura 1 mostra a página inicial da versão anterior e do redesign:

\footnotetext{
${ }^{7}$ https://bit.ly/3CdQNdY

${ }^{8}$ https://bit.ly/2VozA1A
} 


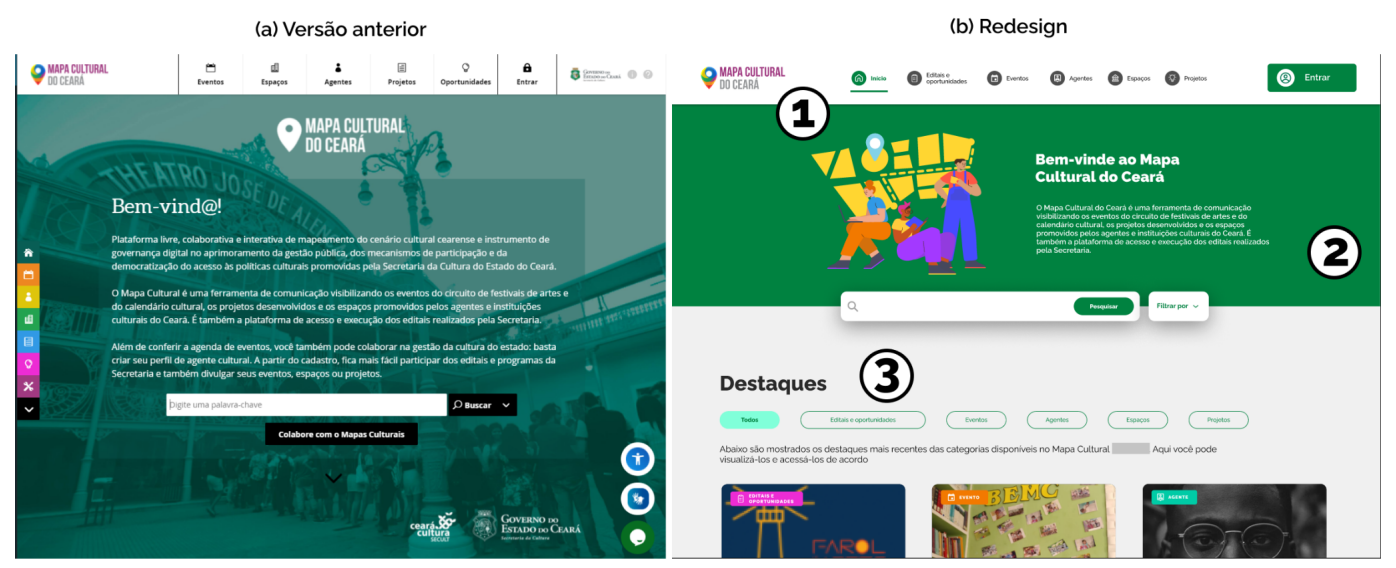

Figura 1. Página inicial da versão anterior (à esquerda) e do redesign (à direita)

Em (1), visualiza-se como problemas de informar status do usuário e destaque de botões foram resolvidos, de modo a deixar em evidência como o usuário pode se guiar pela interface. Em (2) mostra-se que o texto da página inicial foi resumido (foi apontado nos testes que o texto era longo) e também foi proposta uma nova solução visual. Em (3) visualiza-se parte importante da reestruturação da página inicial, disponibilizando acesso rápido a informações inseridas na plataforma.

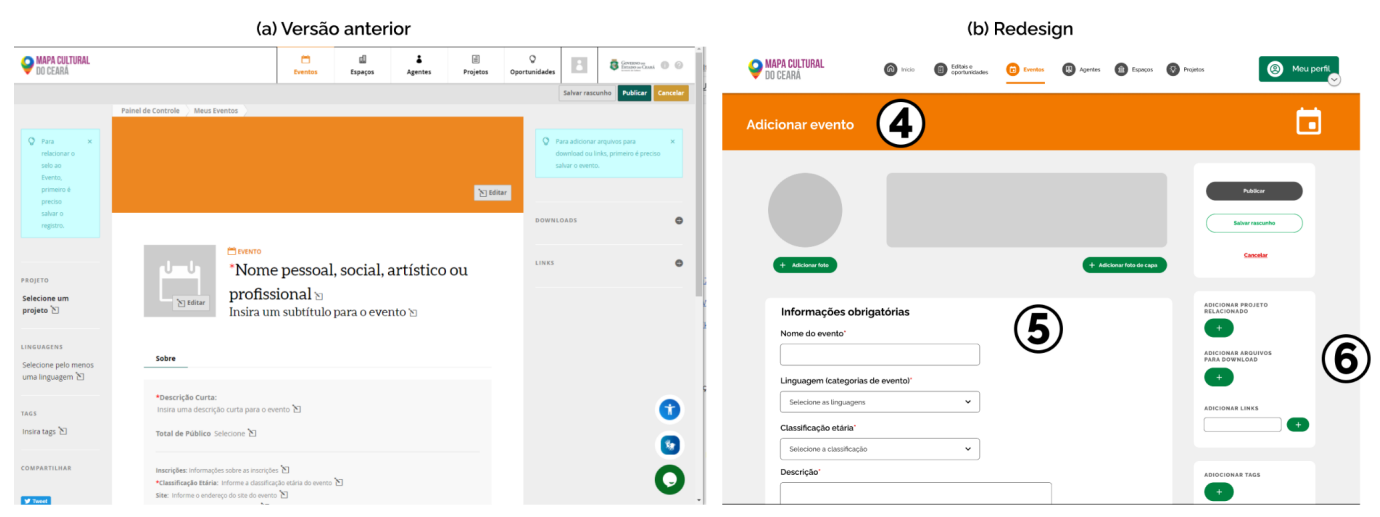

Figura 2. Exemplo de mudanças numa página de formulário

A Figura 2 mostra um comparativo de formulário de adição de eventos entre as versões. Em (4) evidencia-se a informação do status do usuário principalmente para o título da seção mais destacado e também pode-se notar que os campos do formulários estão mais evidentes e perceptíveis (5). Em (6) destaca-se o agrupamento de botões e a reestruturação da página, que antes eram separados em três colunas.

\subsection{Avaliação do Redesign}

A etapa de avaliação do redesign ${ }^{9}$ teve como objetivo validar junto aos usuários que aspectos do redesign contribuíram para a percepção de melhorias. Então, foram realizados testes de usabilidade e entrevistas com cinco usuários. Neles, se percebeu que as atividades foram realizadas de forma efetiva registrando apenas 7 erros, relacionados a visibilidade e agrupamento de elementos.

\footnotetext{
${ }^{9}$ https://bit.ly/3AesAUj
} 
Percebeu-se ainda que o redesign reforça a simplicidade de interações que a plataforma já trazia, visto que nos testes iniciais os usuários não tiveram problemas para fazer as atividades. Considerando também a entrevista, se percebeu que os usuários puderam dar mais sugestões em relação ao que sentiam falta do que nos primeiros testes. Nas falas dos entrevistados apareceram expressões como "modernidade", "organização", "simpática", "atrativa", "amigável" e "didática" para descrever o redesign da plataforma. Nesse sentido, os usuários são positivos sobre as mudanças quanto a facilidade de utilização, mas principalmente devido ao visual estético e os elementos gráficos utilizados, demonstrando também que a percepção de melhorias ajuda a estimular o interesse deles em utilizar a plataforma.

\section{Discussão}

Com aspecto colaborativo, o Mapa Cultural do Ceará busca que a maior parte das pessoas utilizadoras sejam participantes ativos na construção das informações e recursos disponibilizados nele. Assim, a seguinte afirmação de um entrevistado "tá bem simplificado os processos! Na antiga, quando você queria criar um evento era muito confuso" indica que o objetivo de melhoria em como os usuários inserem ou editam informações foi atingido ao se melhorar a visibilidade de campos, ao aumentar o tamanho dos botões e textos informativos, organizar os campos em grupos, ao usar mensagens de confirmação e ao agrupar elementos relacionados.

Para estimular a motivação dos usuários também se propôs uma nova identidade visual que melhorasse a utilização de ícones e botões, e fosse mais atrativa aos usuários ao transmitir aspectos de renovação e de atualização. Para isso, também foram utilizadas ilustrações visando uma experiência mais atrativa e amigável com a interface [El-Sherbiny 2020]. A seguinte frase de um usuário "um design bem feito, algo bonito, que tem uma usabilidade bacana estimula a pessoa a usar aquilo", corrobora que a interface agradou os usuários estimulando o desejo de usar por parte dos usuários.

Também destacam-se o processo e os métodos utilizados, à medida em que possibilitaram analisar questões de usabilidade, e questões de experiência do usuário, que para este trabalho se fazem relevantes, pois foi possível entender aspectos sentimentais e as motivações dos usuários para colaborarem com a construção de informações na plataforma. Porém, é importante ressaltar as limitações do trabalho, como a quantidade mínima de usuários (apenas cinco pessoas) ao se avaliar o redesign. Além disso, este projeto teve limitações de tempo que inviabilizaram uma nova avaliação do redesign com maior número de participantes, de modo a dar robustez aos dados encontrados.

\section{Conclusão}

Propôs-se uma nova interface do Mapa Cultural com foco nos pontos descobertos no processo e que melhorou ainda mais a relação dos usuários com o artefato aqui discutido, estimulando a colaboração. Nesse sentido, as maiores dificuldades percebidas foram quanto a limitação de tempo e a adaptação de testes com usuários para um formato remoto. Assim, este trabalho contribui com a discussão sobre os processos centrados no usuário focados em plataformas colaborativas, tendo potencial de auxiliar no desenvolvimento e melhorias de outras plataformas que utilizam a base do projeto 
Mapas Culturais. Trabalhos futuros também podem se debruçar sobre políticas públicas que incentivem o uso da plataforma e a maximização do aspecto social dela, explorando novas possibilidades e interação entre os usuários.

\section{Referências}

Castro, R., Araújo, A. A., Soares, P., Paixao, M., Maia, P. H. M., \& Souza, J. (2021, April). Proposta de Mapa Interativo e Colaborativo para Ecossistemas de Inovação: Um Estudo de Caso Baseado no Estado do Ceará. In Anais do XVII Simpósio Brasileiro de Sistemas Colaborativos (pp. 59-70). SBC.

Cavalcante, E., Rivero, L., \& Conte, T. (2015). MAX: A Method for Evaluating the Post-use User eXperience through Cards and a Board. In 27th International Conference on Software Engineering and Knowledge Engineering (SEKE 2015) (pp. 495-500).

El-Sherbiny, H. (2020). The Use Of Flat Illustrations in Websites and Smartphones Application's User Interface (UI) Design. Journal of Arts \& Architecture Research Studies, 1(2-December 2020), 198-212.

Hassenzahl, M. (2008). User experience (UX) towards an experiential perspective on product quality. In Proceedings of the 20th Conference on l'Interaction Homme-Machine (pp. 11-15).

Secretaria da Cultura do Estado do Ceará. (2015). Mapa Cultural do Ceará. Mapa Cultural do Ceará. https://mapacultural.secult.ce.gov.br

Nascimento, F. P. do (2015). Mapas colaborativos e o espaço público: a utilização de sistemas de mapeamento colaborativo online como ferramenta nos processos de requalificação urbana.

Pribeanu, C. (2017). A Revised Set of Usability Heuristics for the Evaluation of Interactive Systems. Informatica Economica, 21(3).

Ribeiro, J. C. S., \& Lima, L. B. (2011). Mapas colaborativos digitais e (novas) representações sociais do território: uma relação possível. C-Legenda-Revista do Programa de Pós-graduação em Cinema e Audiovisual, (25).

Rigolon, T., Mazzonetto, C., Mubarack, H., \& Tolezano, A. C. (2018). Painel Mapas Culturais: Uma experiência de análise de dados. Revista do Centro de Pesquisa e Formação, pp. 21-38.

Rigolon, T. (2016). Mapas Culturais. Congreso Online de Gestión Cultural, pp. 1-5.

Rogers, Y., Sharp, H., \& Preece, J. (2005). Design de interação. Bookman Editora.

Steil, C. (2018). Requisitos para o redesign da RedeParaná: uma abordagem centrada no usuário.

Vermelho, S. C. Velho, A. P. M., Bonkovoski, A., \& Pirola, A. (2014). Refletindo sobre as redes sociais digitais. Educação \& sociedade, 35, 179-196. 